\title{
A new risk variant for multiple sclerosis at the immunoglobulin heavy chain locus associates with intrathecal IgG, IgM index and oligoclonal bands
}

\author{
Mercedes Delgado-García, Fuencisla Matesanz, Antonio Alcina, María Fedetz, María Isabel \\ García-Sánchez, Juan Luis Ruiz-Peña, Óscar Fernández, María Jesús Pinto Medel, Laura Leyva, \\ Carmen Arnal, Concepción Delgado, José Antonio López Guerrero, Antonio González-Pérez, \\ María E Sáez, Luisa María Villar, José Carlos Álvarez-Cermeño, Carmen Picón, Rafael Arroyo, \\ Jezabel Varadé, Elena Urcelay, Guillermo Izquierdo and Miguel Lucas
}

\section{Abstract}

Background: Recent findings have shown a correlation between the intrathecal IgG index and variants at the immunoglobulin heavy chain (IGHC) locus in patients with multiple sclerosis (MS).

Objectives: The objective of this paper is to analyse the association of the locus with MS susceptibility and its relationship with intrathecal immunoglobulin (Ig) parameters.

Methods: We genotyped the rs11621145 variant, located at the IGHC locus, in 2726 patients with MS and 2133 healthy controls. Associations of intrathecal IgG and IgM indexes with rs11621145 were analysed by linear regression analysis in 538 MS patients.

Results: We found that rs 11621145 showed statistically significant evidence for association with susceptibility to MS (odds ratio $=0.69, p=1.053 \mathrm{E}-09$ ), though validation of this result in additional cohorts would be desirable. We confirmed the association between the IgG index and the rs11621145 ( $p=6.85 \mathrm{E}-$ 07 , Beta $=0.207)$. Furthermore, rs 11621145 was inversely correlated with $\operatorname{IgM}$ index $(p=7.24 \mathrm{E}-04$, Beta $=-0.277$ ), and therefore marks a decreased likelihood of presenting IgM oligoclonal bands (odds ratio $=$ $0.38, p=2.35 \mathrm{E}-06)$.

Conclusions: Our results suggest that the polymorphism of the IGHC locus could be altering the switching of the Ig isotype in B cells and it may be interfering with T-dependent and T-independent antibody responses.

Keywords: Multiple sclerosis (MS), intrathecal $\operatorname{IgG} / \operatorname{IgM}$ index, oligoclonal bands (OCBs), immunoglobulin heavy chain (IGHC) locus, association, SNP variant.

Date received: 1 April 2014; revised: 14 July 2014; 8 September 2014; accepted: 28 September 2014

\section{Introduction}

Multiple sclerosis (MS) is the most frequent demyelinating disease of the central nervous system (CNS) with an active inflammatory and immune component. ${ }^{1,2}$ MS is widely accepted as an autoimmune disease, but the aetiology and pathogenic mechanisms remain unclear. Current knowledge supports that MS develops in genetically susceptible individuals as a result of an autoimmune attack to the myelin sheath activated through environmental factors. ${ }^{3}$ In MS, activated CD4+ T cells enter the CNS and trigger an inflammatory cascade that leads to recruitment of other immune cells such as B, $\mathrm{CD} 8+\mathrm{T}$ cells and macrophages and induces the production of cytokines, antibodies and complement factors.
Multiple Sclerosis Journal

$1-8$

DOI: $10.1177 /$

1352458514556302

(C) The Author(s), 2014 Reprints and permissions: http://www.sagepub.co.uk/ journalsPermissions.nav
Correspondence to: Fuencisla Matesanz Departamento de Biología Celular e Inmunología, Instituto de Parasitología y Biomedicina López Neyra (IPBLN), Consejo Superior de Investigaciones Científicas (CSIC), 18016 Granada,

Spain

lindo@ipb.csic.es

María Isabel García-Sánchez Juan Luis Ruiz-Peña Unidad de Esclerosis

Múltiple, Hospital

Universitario Virgen Macarena, Sevilla, Spain

Mercedes Delgado-García Guillermo Izquierdo Unidad de Esclerosis Múltiple, Hospital Universitario Virgen Macarena, Sevilla, Spain/ These authors contributed equally to this work

Antonio Alcina Maria Fedetz Departamento de Biología Celular e Inmunología, Instituto de Parasitología y Biomedicina López Neyra (IPBLN), Consejo Superior de Investigaciones Científicas CSIC, 18016 Granada, Spain Fuencisla Matesanz Departamento de Biología Celular e Inmunología, Instituto de Parasitología y Biomedicina López Neyra (IPBLN), Consejo Superior de Investigaciones Científicas CSIC, 18016 Granada, Spain These authors contributed equally to this work

Óscar Fernández María Jesús Pinto Medel Laura Leyva Unidad de Gestión Clínica de Neurociencias, Instituto de Biomedicina de Málaga (IBIMA), Hospital Regional Universitario de Málaga, Málaga, Spain 
Carmen Arnal

Servicio de Neurología,

Hospital Virgen de las

Nieves, Granada, Spain

Concepción Delgado

Centro Regional de

Transfusión Sanguínea

Granada-Almería, Ganada,

Spain

José Antonio López

Guerrero

Centro de Biología Molecula

Severo Ochoa, CSIC-UAM,

Madrid, Spain

Antonio González-Pérez

María E Sáez

Centro Andaluz de Estudios

Bioinformáticos (CAEBi),

Sevilla, Spain

Luisa María Villar

José Carlos Álvarez-

Cermeño

Carmen Picón

Hospital Ramon y Cajal,

Departments of Immunology

and Neurology, MS Unit,

(IRYCIS), Madrid, Spain

Rafael Arroyo

Multiple Sclerosis Unit,

Hospital Clínico San Carlos,

Instituto de Investigación

Sanitaria del Hospital Clínico

San Carlos (IdISSC), Madrid,

Spain

Jezabel Varadé

Elena Urcelay

ImmunologyDepartment,

Hospital Clínico San Carlos,

Instituto de Investigación

Sanitaria del Hospital Clínico

S. Carlos (IdISSC), Madrid,

Spain

Miguel Lucas

Servicio de Biologí

Molecular, Hospital

Universitario Virgen

Macarena, Facultad de

Medicina, Sevilla, Spain

These authors contributed

equally to this work
The role of cerebrospinal fluid (CSF)-resident B cells seems to be important in CNS inflammation, but their contribution to MS evolution and progression is less clear. ${ }^{4}$ Nonetheless, antigen-directed affinity maturation and terminal differentiation of $\mathrm{B}$ cells contributing to $\mathrm{IgG}$ secretion and $\mathrm{IgG}$ oligoclonal bands (OCBs) occurs within perivascular infiltrates and meningeal lymphoid-like follicles in the CNS. 5,6

An elevation of intrathecal IgG synthesis and the presence of OCBs, characteristics of MS, ${ }^{7}$ further suggest the autoimmune origin of lesions in MS. In fact, about $95 \%$ of patients with MS show IgG-OCBs, and close to $40 \%$ show IgM OCBs in CSF. ${ }^{8,9}$ Moreover, it has been reported that intrathecal IgM synthesis predicts a worse disease course in the first stages of MS. ${ }^{10}$ Analysis of the $\mathrm{IgG}$ index, which is elevated in about $70 \%$ of patients, as well as the presence of $\mathrm{OCBs}^{11,12}$ have been proposed as complementary data for the diagnosis of MS. ${ }^{13}$

Buck et al. ${ }^{14}$ have recently reported that genetic variants in the immunoglobulin heavy chain (IGHC) locus are associated with the $\operatorname{IgG}$ index in $\mathrm{MS}$, and that specific haplotypes might affect intrathecal IgG synthesis.

We aimed to study the genetic association as well as exploring the immunoglobulin (Ig) profile and OCBs in a cohort of Spanish patients with MS. Part of this cohort was included in a recent genome-wide association (GWA) study. ${ }^{15}$ Here, we recruited additional patients from the same study site and from two other Spanish centres, attaining 2726 MS patients and 2133 sex- and age-matched controls. In this work, we investigate the association with MS of single nucleotide polymorphisms (SNPs) within the IGHC locus and their relationship with the intrathecal $\operatorname{IgG}$ and IgM synthesis, as well as with the presence of OCBs for both Igs. In addition to confirming the findings of Buck et al. ${ }^{14}$ regarding the association of the $I G H C$ locus with the IgG index, we observed that the selected variant was associated with both MS susceptibility and $\operatorname{IgM}$ status.

\section{Patients and methods}

\section{Patients}

Patients with suspicion of MS undergo lumbar puncture as part of the diagnostic procedure. Diagnosis was made by a neurologists based on McDonald criteria. ${ }^{16}$ After diagnosis patients were classified as clinically isolated syndrome (CIS), clinically definite MS, or non-MS patients. The study cohort from Hospital
Universitario Virgen Macarena (Sevilla) consisted of 362 patients with MS, and 414 patients free of MS or any other inflammatory disease (i.e. suffering mainly from headaches, hydrocephalus, Alzheimer's disease, Parkinson, behavioural changes, and stroke). The cohort from Hospital Ramón y Cajal (Madrid) consisted of 179 MS patients. Main features of the patients are shown in Table 1. Patients provided informed consent for lumbar puncture.

The entire cohort for rs11621145 genotyping study comprised 2726 patients with MS meeting established diagnostic criteria ${ }^{16}$ and 2133 healthy controls (mostly blood donors and staff). All patients and healthy controls had Caucasian origin. Patients and controls were recruited from six different Spanish hospitals: Sevilla cohort consisting of 988 cases and 1070 controls from Hospital Virgen Macarena, Hospital Virgen de la Nieves and Blood Bank of Andalucía; Málaga cohort consisting of 647 cases and 402 controls from Hospital Carlos Haya; Madrid cohort consisting of 1091 cases and 661 controls from Hospital Clínico San Carlos and Hospital Ramón y Cajal. Patients provided informed consent for blood extraction. The institutional ethics committees of these centres approved the study.

\section{Samples and OCB studies}

CSF samples were obtained by lumbar puncture from patients under suspicion of MS. The study of OCBs was performed in laboratories from Hospital Universitario Virgen Macarena (Seville) and Ramón y Cajal (Madrid). The procedure included the two-step detection of OCBs by isoelectric-focusing and incubation with antibodies to either $\operatorname{IgG}$ or $\operatorname{IgM} .{ }^{17,18} \operatorname{Ig}$ and albumin concentration in CSF and serum were measured by nephelometry. We quantified intrathecal IgG and IgM synthesis as the Ig index (CSF/serum Ig ratio divided by the CSF/serum albumin ratio). ${ }^{11}$ These determinations are included in the protocol of routine diagnosis for MS in the above-indicated hospitals.

\section{SNP selection and genotyping}

SNP selection was based on the SNPs described by Buck et al. ${ }^{14}$ located at chromosome 14, and associated with $\mathrm{IgG}$ index. Linkage disequilibrium and Hardy-Weinberg (HW) equilibrium was performed with the 1000 Genome Project data for Caucasian populations. ${ }^{19}$ DNA was extracted from peripheral blood cells by standard methods using either the Maxwell Kit (Promega) or a saline precipitation procedure. The SNP rs11621145 was genotyped using Taqman ${ }^{\circledR}$ SNP Genotyping Assays (Applied Biosystems) according 
Table 1. Demographic, clinical characteristics and cerebrospinal fluid parameters of the studied cohorts.

\begin{tabular}{|c|c|c|c|c|c|c|}
\hline \multirow[t]{2}{*}{ Cohorts } & \multicolumn{2}{|l|}{ Madrid } & \multicolumn{2}{|c|}{ Málaga } & \multicolumn{2}{|l|}{ Sevilla } \\
\hline & MS & Control & MS & Control & MS & Control \\
\hline \multicolumn{7}{|l|}{ MS association study } \\
\hline$N$ & 1091 & 661 & 647 & 402 & 988 & 1070 \\
\hline Age, mean year & 45 & 48 & 45 & 42 & 42 & 41 \\
\hline Female (\%) & 54 & 66 & 67.7 & 67 & 67 & 67 \\
\hline PP $(\%)$ & 4.2 & & 1.4 & & 6.3 & \\
\hline \multicolumn{7}{|l|}{ Ig Index study } \\
\hline$N$ & 179 & & & & 362 & \\
\hline Age, mean year at puncture (range) & $37(13-69)$ & & & & $35(12-72)$ & \\
\hline Females (\%) & 70 & & & & 70 & \\
\hline IgG index, geometric mean (range) & $1(0.28-3.77)$ & & & & $0.96(0.41-6.63)$ & \\
\hline IgM index, geometric mean (range) & $0.19(0.02-2.86)$ & & & & $0.17(0.01-5.53)$ & \\
\hline IgG OCB total / + (\%) & $151 / 129(85)$ & & & & $491 / 456(93)$ & \\
\hline IgM OCB total $/+(\%)$ & $182 / 77(42)$ & & & & $257 / 112(44)$ & \\
\hline
\end{tabular}

to the manufacturer's specifications. Genotyping call rates were $>95 \%$ in each cohort analysed and $10 \%$ of the samples were genotyped in duplicate with $>99 \%$ concordance.

\section{Statistical analysis}

Departure from HW equilibrium was tested using an exact test. Genotype data were analysed to obtain allele and genotype counts using PLINK V.1.07. ${ }^{20}$ For each available cohort odds ratios (OR), 95\% Confidence Intervals (CI) and $p$-value were estimated by fitting sex-adjusted logistic regression models with PLINK in order to assess the associations between rs11621145 and: (a) the MS risk; (b) the presence of IgM OCB; and (c) the presence of IgG OCB. Both additive and dominant models were explored. In order to assess the association between Ig indexes and rs11621145, both indexes were transformed using natural logarithms as previously done by Buck et al. ${ }^{14}$ and, as happened in their study, untransformed data did not follow a Gaussian distribution, but was rightskewed. Once transformed, we analysed both IgG and IgM indexes by using the "-linear" command in PLINK that fits a linear regression. Thus, for each available cohort we obtained sex and age of punctureadjusted beta coefficients, $95 \% \mathrm{CI}$, and $p$-values using both additive and dominant models. Estimates across cohorts were obtained by running meta-analyses for each endpoint using the "metan" command in Stata 12 (College Station, TX, USA). Both random and fixed-effects models were used and heterogeneity was explored by estimating $I^{2}$ index and $Q$ statistic.

\section{Results}

\section{$\operatorname{Ig} G$ index associated variant is a risk factor for $M S$}

Buck et al. ${ }^{14}$ reported five SNPs at the Ig locus associated with IgG index in MS patients by GWA analysis. Among these variants, the most associated was rs10136766. These polymorphisms present high linkage disequilibrium (LD) as determined in 379 Caucasian individuals included in the 1000 Genomes Project $\left(r^{2}>0.49-0.95\right)$. However, this polymorphism is not in HW equilibrium according to the 1000 Genomes Project data. In fact, only the second-most associated variant, rs11621145, was found in HW equilibrium, and therefore we selected this SNP as the best candidate to assess the association of this locus with IgG index and MS susceptibility.

As shown in Table 2, rs11621145 was genotyped in three Spanish cohorts whose controls were in HW equilibrium. After logistic regression analyses, we found that the dominant model was the one that best fitted the data. The rs 11621145 was associated to MS in all three cohorts, showing a protective effect among $\mathrm{G}$ allele carriers. The meta-analysis of these results along with those from Buck et al. ${ }^{14}$ for rs 11621145 is also indicated (Figure 1).

This locus has not been previously associated with MS in any of the GWA studies performed so far, but the coverage of this locus in the chips used is very low. 

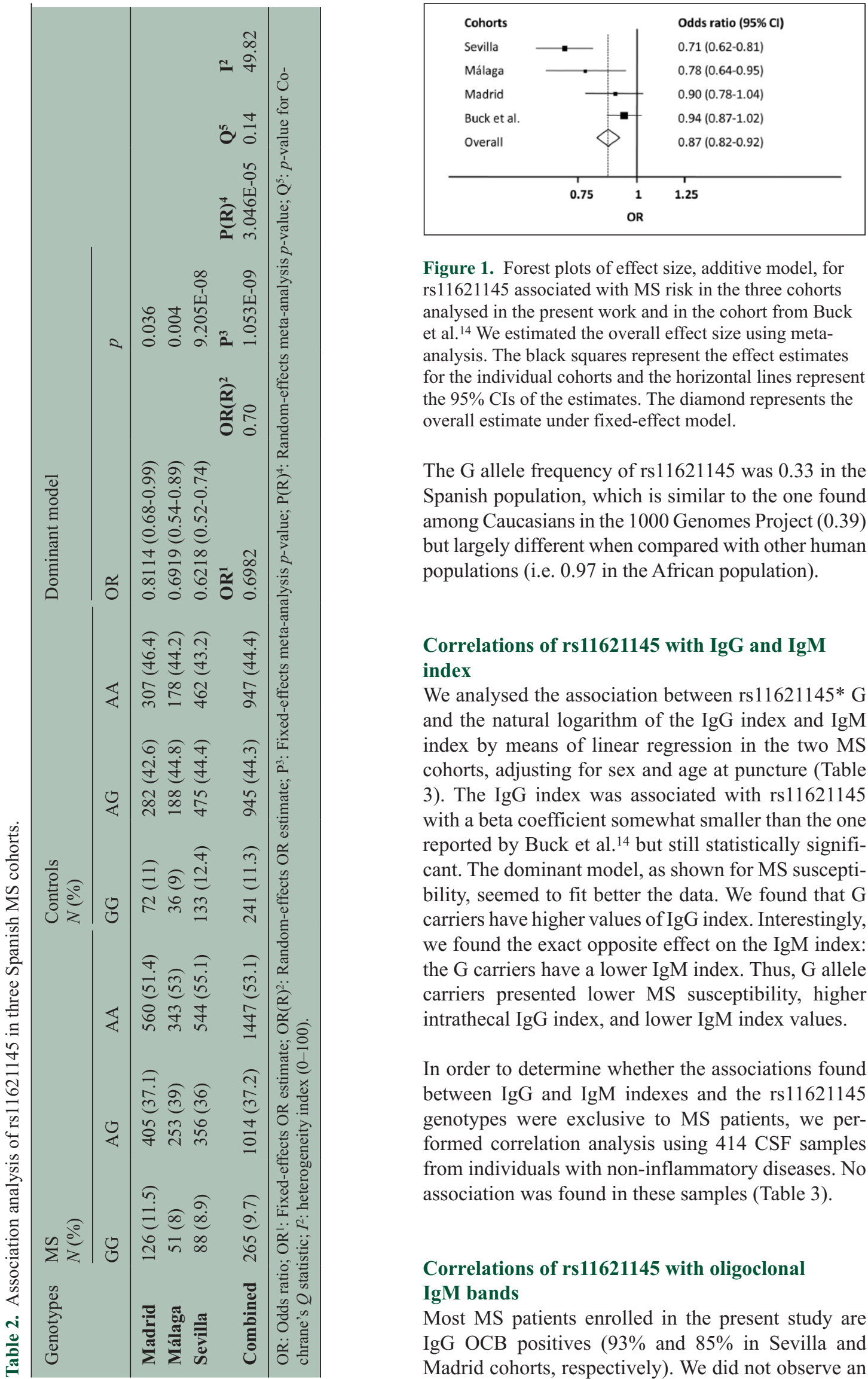

Figure 1. Forest plots of effect size, additive model, for rs11621145 associated with MS risk in the three cohorts analysed in the present work and in the cohort from Buck et al. ${ }^{14}$ We estimated the overall effect size using metaanalysis. The black squares represent the effect estimates for the individual cohorts and the horizontal lines represent the $95 \%$ CIs of the estimates. The diamond represents the overall estimate under fixed-effect model.

The G allele frequency of rs 11621145 was 0.33 in the Spanish population, which is similar to the one found among Caucasians in the 1000 Genomes Project (0.39) but largely different when compared with other human populations (i.e. 0.97 in the African population).

\section{Correlations of rs11621145 with IgG and IgM index}

We analysed the association between rs $11621145^{*} \mathrm{G}$ and the natural logarithm of the $\operatorname{IgG}$ index and $\operatorname{IgM}$ index by means of linear regression in the two MS cohorts, adjusting for sex and age at puncture (Table 3). The IgG index was associated with rs 11621145 with a beta coefficient somewhat smaller than the one reported by Buck et al. ${ }^{14}$ but still statistically significant. The dominant model, as shown for MS susceptibility, seemed to fit better the data. We found that $G$ carriers have higher values of IgG index. Interestingly, we found the exact opposite effect on the IgM index: the $\mathrm{G}$ carriers have a lower IgM index. Thus, $\mathrm{G}$ allele carriers presented lower MS susceptibility, higher intrathecal $\operatorname{IgG}$ index, and lower IgM index values.

In order to determine whether the associations found between $\operatorname{IgG}$ and $\operatorname{IgM}$ indexes and the rs 11621145 genotypes were exclusive to MS patients, we performed correlation analysis using 414 CSF samples from individuals with non-inflammatory diseases. No association was found in these samples (Table 3 ).

\section{Correlations of rs11621145 with oligoclonal IgM bands}

Most MS patients enrolled in the present study are IgG OCB positives $(93 \%$ and $85 \%$ in Sevilla and Madrid cohorts, respectively). We did not observe an 
Table 3. Association study of the IgG and IgM index from MS and No Inflammatory Disease patients with the rs11621145 SNP by linear regression analysis.

\begin{tabular}{|c|c|c|c|c|c|c|c|c|c|c|c|}
\hline & & & \multicolumn{3}{|c|}{ Sevilla } & \multicolumn{3}{|c|}{ Madrid } & \multicolumn{3}{|c|}{ Meta-analysis } \\
\hline & & & $N$ & BETA & $p$ & $N$ & BETA & $p$ & $N$ & BETA & $p$ \\
\hline \multirow{5}{*}{ MS } & \multirow[t]{2}{*}{ IgG } & $\mathrm{ADD}$ & 362 & 0.147 & $1.42 \mathrm{E}-04$ & 176 & 0.101 & 0.066 & 538 & 0.132 & $2.52 \mathrm{E}-05$ \\
\hline & & DOM & 362 & 0.215 & $2.6 \mathrm{E}-05$ & 176 & 0.187 & 0.014 & 538 & 0.207 & $6.85 \mathrm{E}-07$ \\
\hline & & & & & & & & & & & \\
\hline & \multirow[t]{2}{*}{ IgM } & $\mathrm{ADD}$ & 292 & -0.219 & 0.007 & 173 & -0.227 & 0.013 & 465 & -0.223 & $2.2 \mathrm{E}-04$ \\
\hline & & DOM & 292 & -0.295 & 0.0063 & 173 & -0.252 & 0.048 & 465 & -0.277 & $7.24 \mathrm{E}-04$ \\
\hline & IgG & $\mathrm{ADD}$ & 414 & 0.003 & 0.73 & & & & & & \\
\hline & & DOM & 414 & 0.007 & 0.57 & & & & & & \\
\hline \multicolumn{12}{|l|}{ No MS } \\
\hline & \multirow[t]{2}{*}{ IgM } & $\mathrm{ADD}$ & 414 & 0.003 & 0.73 & & & & & & \\
\hline & & DOM & 414 & 0.0064 & 0.92 & & & & & & \\
\hline
\end{tabular}

Table 4. Association between the rs11621145 SNP and the IgM OCB.

\begin{tabular}{|c|c|c|c|c|c|c|}
\hline & \multirow[t]{2}{*}{ Allele } & \multirow[t]{2}{*}{$N$} & Dominant model & \multirow[t]{2}{*}{$p$} & Additive model & \multirow[t]{2}{*}{$p$} \\
\hline & & & OR $(95 \% \mathrm{CI})$ & & OR $(95 \% \mathrm{CI})$ & \\
\hline Sevilla & G & 255 & $0.43(0.26-0.72)$ & 0.0014 & $0.4937(0.33-0.75)$ & 0.0008 \\
\hline Madrid & $\mathrm{G}$ & 179 & $0.32(0.17-0.6)$ & 0.00038 & $0.4102(0.25-0.67)$ & 0.00033 \\
\hline Meta-Analysis & & & 0.3827 & $2.35 \mathrm{E}-06$ & 0.4569 & $1.08 \mathrm{E}-06$ \\
\hline
\end{tabular}

association between rs11621145 genotypes and $\mathrm{IgG}$ OCB. However, the power of this analysis was limited due to the low number of IgG OCB-negative patients, thus we cannot discard this association. On the other hand, we did observe an association between the presence of IgM OCB and the rs11621145 variant in both cohorts available (Sevilla and Madrid) (Table 4). In this case the additive model seemed to fit better the results. The $\mathrm{G}$ allele was associated with a lower likelihood of presenting IgM OCBs. The $\mathrm{G}$ allele was associated with protection towards MS, higher IgG index, lower IgM index and absence of IgM OCBs.

\section{Discussion}

In this work we confirm the previously reported association of the IGHC locus with the intrathecal IgG index in MS. ${ }^{14}$ In addition, we have found that this association does not only involve the $\mathrm{IgG}$ but also the IgM index and IgM OCB. The opposite effects observed in the associations between the IgG and IgM indexes with the SNP under study, along with the localization of this polymorphism in the IGHC gene locus, point to an alteration of the equilibrium between innate and T-associated immune responses as the functional cause of the association.

In the T-cell-mediated immune response, IgM-positive B lymphocytes switch to IgG, IgA, or IgE upon immunization or infection. ${ }^{22}$ This process occurs by a recombination within $\mathrm{S}$ regions located upstream of each one of the heavy chain constant region $\left(\mathrm{C}_{\mathrm{H}}\right)$ genes resulting in a change from $\operatorname{IgM}$ expression to one of the downstream isotypes. The isotype switching is cytokinedirected by inducing transcription from promoters located upstream to each acceptor $\mathrm{S}$ region. Cisregulatory elements have been described within the $I G H C$ gene locus and participate in the regulation of Ig $\mathrm{C}_{\mathrm{H}}$ gene expression and isotype switch. Different knockout experiments have demonstrated the importance of $\mathrm{C}_{\mathrm{H}}$ gene transcription for efficient isotype switch. ${ }^{23-25}$ In fact, a human polymorphism in the regulatory region located at the 3 ' of the IGHC gene locus, 3'RR enhancer, regulates $\mathrm{C}_{\mathrm{H}}$ gene transcription. ${ }^{26}$ This 
polymorphism associates with selective IgA deficiency (IGAD), a common primary immunodeficiency, and with IgM concentration. ${ }^{27}$ Variants in the same 3 'RR enhancer have been also associated with systemic lupus erythematosus. ${ }^{28}$ In the innate antibody response, responsible for the production of more than $80 \%$ of serum IgM, antibodies are produced against philogenetically conserved antigens predominantly of nonprotein nature. ${ }^{29}$ This antibody response is mainly produced by $\mathrm{CD} 5+\mathrm{B}$ cells and is responsible for intrathecal IgM synthesis in MS. ${ }^{30}$

The polymorphisms associated with IgG index reported by Buck et al. ${ }^{14}$ and validated in this work are located in the immunoglobulin gamma heavy chain genes $(I G H G)$ region, where four IGHG are encoded. However, since we do not know the ultimate causal variant of the Ig index alteration, it is not possible to infer whether the studied variant may be affecting cis-regulatory elements located upstream of the heavy chain gene modifying expression or whether it may be interfering with other process during class switching; further study in this area must be performed to clarify the underlying mechanism. It is noteworthy that the association between Ig index and rs11621145 occurs exclusively among MS patients and not in patients with non-inflammatory diseases. However, in these control patients, the intrathecal IgG would originate from the periphery. It would be important to analyse the $\operatorname{IgG}$ levels in a group of patients with diseases where intrathecal IgG synthesis occurs, to determine if the observed effect of the polymorphism on the Ig levels is specific to MS. The inflammatory environment of the brain in patients with MS could be the circumstance that triggers the observed effect. Cytokines produced by $\mathrm{T}$ helper and antigen-presenting cells determine the isotype to which B cells will switch, by inducing transcription from promoters located upstream to each acceptor $\mathrm{S}$ region. The promoters for the four $I G H G$ are differentially regulated. They do not respond equally to regulatory stimulus, as has been shown by their differential response to the deletion of the Ikaros transcription factor in an animal model. ${ }^{31}$ In fact, there are several works pointing to the production of specific IgG isotypes in the CSF of patients MS. ${ }^{32,33}$ The specific cytokine spectrum produced in the brain of the MS patient could induce the switch to mainly one of the $I G H G$ and this process could be altered by the polymorphism.

In this work we showed an association of the Ig locus with MS. However, our results were contradictory to those previously reported by Buck et al., ${ }^{14}$ which reported a negative association of the locus with MS.
Even though in the present study we reached a large number of patients and controls from the meta-analysis between Spanish and UK cohorts, we should not forget that the small effects of non-HLA loci associated with MS require very large cohorts to demonstrate an association with the disease. On the other hand, even though all patients and controls were of Caucasian origin from Spain, we cannot totally rule out that residual population stratification could be partially responsible for the disease association observed here. For these reasons, it would be desirable for other groups to replicate this association, to reach numbers as reported in studies such as the Immunochip Project, ${ }^{34}$ to completely overcome any doubts about its involvement in MS susceptibility.

The differences of association observed between cohorts were not unexpected, since previous metaanalyses have reported similar situations. ${ }^{34}$ However, in this case we found notable differences between Sevilla and the rest of the cohorts. One clear difference of this cohort is that almost all patients were $\operatorname{IgG}$ OCB positive. We believe this could be one of the possible reasons for the observed differences in MS association between cohorts.

The homozygote individuals for the major allele (A) show an increased MS risk. The A allele is also associated with higher IgM index, higher IgM OCB and lower IgG index. One may think that since $A$ is the major allele, the basal function should be associated with this allele, and $\mathrm{G}$ carriers might have acquired a higher ratio of class switching. However, when we analysed the allele frequency of rs11621145 in human populations other than Europeans, we observed that the A allele is almost non-existent in African populations. Since we do not know the ultimate causal variant of the effect, we cannot establish whether among African populations the rs 11621145 polymorphism would not capture the functional variant, or that the polymorphism causing the effect in the Ig index was acquired later in human evolution and conserved in non-African populations.

The ultimate reason why these effects on the intrathecal Ig index and IgM bands can contribute to MS risk deserves further investigation. However, antibody isotype is important for determining the effector antibodies. The $\mathrm{C}_{\mathrm{H}}$ region is bound by the $\mathrm{Fc}$ receptors on many cell types. Different $\mathrm{C}_{\mathrm{H}}$ regions have different affinities for these proteins, thus greatly influencing antibody function and determining whether antibodyantigen complexes will activate cells. Pathological and clinical studies implicate antibody-dependent mechanisms in the immunopathogenesis of MS. 
Therefore, any effect on the antibody isotype can affect MS susceptibility.

\section{Acknowledgements}

We thank the patients with multiple sclerosis and control subjects for making this study feasible. We also acknowledge the University Hospital Virgen Macarena Biobank (Andalusian Public Health System Biobank), integrated in the Spanish Biobanks Network (Carlos III Health Institute, Grant $n^{\circ}$ RD09/0076/00080), for its help and support in the gifts of clinical samples, used in this work.

\section{Conflicts of interest}

No conflicts of interest

\section{Funding}

Financial support for the study was provided by Fondo de Investigación Sanitaria (FIS)-Fondos Europeos de Desarrollo Regional (FEDER) [grant numbers RETICS-REEM-RD12/0032/0015-RD12/0032/0005RD12/0032/0006-RD12/0032/0009, P12/00555, PI10/01616, PI13/01527, PI13/ 01466, CP10/00526, PI12-00239]; Junta de Andalucía- Fondos Europeos de Desarrollo Regional (FEDER) [grant numbers P07CVI-02551, P09-CTS-5218]. MDG is holder of a Neuroinvest fellowship.

\section{References}

1. McFarland HF and Martin R. Multiple sclerosis: A complicated picture of autoimmunity. Nat Immunol 2007; 8: 913-919.

2. Zettl UK, Stüve O and Patejdl R. Immune-mediated CNS diseases: A review on nosological classification and clinical features. Autoimmun Rev 2012; 11 $167-173$

3. Sospedra M and Martin R. Immunology of multiple sclerosis. Annu Rev Immunol 2005; 23: 683-747.

4. Kuenz B, Lutterotti A, Ehling R, et al. Cerebrospinal fluid B cells correlate with early brain inflammation in multiple sclerosis. PLOS ONE 2008; 3: e2559.

5. Prineas JW. Multiple sclerosis: Presence of lymphatic capillaries and lymphoid tissue in the brain and spinal cord. Science 1979; 203: 1123-1125.

6. Serafini B, Rosicarelli B, Magliozzi R, et al. Detection of ectopic B-cell follicles with germinal centers in the meninges of patients with secondary progressive multiple sclerosis. Brain Pathol 2004; 14 164-174.

7. Disanto G, Morahan JM, Barnett MH, et al. The evidence for a role of $\mathrm{B}$ cells in multiple sclerosis. Neurology 2012; 78: 823-832.
8. Kostulas VK, Link H and Lefvert AK. Oligoclonal IgG bands in cerebrospinal fluid. Principles for demonstration and interpretation based on findings in 1114 neurological patients. Arch Neurol 1987; 44: 1041-1044.

9. Villar LM, Masjuan J, González-Porqué P, et al. Intrathecal IgM synthesis in neurologic diseases: Relationship with disability in MS. Neurology 2002; 58: 824-826.

10. Villar LM, Masjuan J, González-Porqué P, et al. Intrathecal IgM synthesis is a prognostic factor in multiple sclerosis. Ann Neurol 2003; 53: 222-226.

11. Lefvert AK and Link H. IgG production within the central nervous system: A critical review of proposed formulae. Ann Neurol 1985; 17: 13-20.

12. Cowdrey GN, Tasker PJ, Gould BJ, et al. Isoelectric focusing in an immobilized $\mathrm{pH}$ gradient for the detection of intrathecal $\mathrm{IgG}$ in cerebrospinal fluid: sensitivity and specificity for the diagnosis of multiple sclerosis. Ann Clin Biochem 1993; 30: 463-468.

13. Petereit HF and Heiss WD. New diagnostic criteria for multiple sclerosis. Ann Neurol 2002; 51: 533-534.

14. Buck D, Albrecht E, Aslam M, et al. Genetic variants in the immunoglobulin heavy chain locus are associated with the IgG index in multiple sclerosis. Ann Neurol 2013; 73: 86-94.

15. Matesanz F, González-Pérez A, Lucas M, et al. Genome-wide association study of multiple sclerosis confirms a novel locus at 5p13.1. PloS One 2012; 7 : e36140.

16. Polman $\mathrm{CH}$, Reingold SC, Banwell B, et al. Diagnostic criteria for multiple sclerosis: 2010 revisions to the McDonald Criteria. Ann Neurol 2011; 69: 292-302.

17. Sádaba MC, González Porqué P, Masjuan J, et al. An ultrasensitive method for the detection of oligoclonal IgG bands. J Immunol Methods 2004; 284: 141-145.

18. Villar LM, González-Porqué P, Masjuán J, et al. A sensitive and reproducible method for the detection of oligoclonal IgM bands. J Immunol Methods 2002; 258: 151-155.

19. 1000 Genomes Project Consortium, Abecasis GR, Altshuler D, et al. A map of human genome variation from population-scale sequencing. Nature 2010; 467: 1061-1073.

20. Purcell S, Neale B, Todd-Brown K, et al. PLINK: A tool set for whole-genome association and populationbased linkage analyses. Am J Hum Genet 2007; 81: 559-575.

21. Sawcer S, Hellenthal G, Pirinen M, et al. Genetic risk and a primary role for cell-mediated immune 
Visit SAGE journals online http://msj.sagepub.com

(SAGEjournals mechanisms in multiple sclerosis. Nature 2011; 476: 214-219.

22. Stavnezer J, Guikema JE and Schrader CE. Mechanism and regulation of class switch recombination. Annu Rev Immunol 2008; 26 : 261-292.

23. Jung S, Rajewsky K and Radbruch A. Shutdown of class switch recombination by deletion of a switch region control element. Science 1993; 259: 984-997.

24. Zhang J, Bottaro A, Li S, et al. A selective defect in IgG2b switching as a result of targeted mutation of the I gamma $2 \mathrm{~b}$ promoter and exon. EMBO J 1993; 12: $3529-3537$.

25. Oruc $\mathrm{Z}$, Boumédiène $\mathrm{A}$, Le Bert $\mathrm{M}$, et al. Replacement of Igamma3 germ-line promoter by Igamma1 inhibits class-switch recombination to IgG3. Proc Natl Acad Sci U S A 2007; 104: 20484-20489.

26. $\mathrm{Hu}$ Y, Pan Q, Pardali E, et al. Regulation of germline promoters by the two human Ig heavy chain 3' alpha enhancers. J Immunol 2000; 164: 6380-6386.

27. Giambra V, Cianci R, Lolli S, et al. Allele *1 of HS1.2 enhancer associates with selective IgA deficiency and IgM concentration. J Immunol 2009; 183: 8280-8285.

28. Frezza D, Tolusso B, Giambra V, et al. Polymorphisms of the IgH enhancer HS1.2 and risk of systemic lupus erythematosus. Ann Rheum Dis 2012; 71: 1309-1315.

29. Boes M, Prodeus AP, Schmidt T, et al. A critical role of natural immunoglobulin $\mathrm{M}$ in immediate defense against systemic bacterial infection. J Exp Med 1998; 188: 2381-2386.

30. Villar LM, Sádaba MC, Roldán E, et al. Intrathecal synthesis of oligoclonal IgM against myelin lipids predicts an aggressive disease course in MS. J Clin Invest 2005; 115: 187-194.

31. Sellars M, Reina-San-Martin B, Kastner P, et al. Ikaros controls isotype selection during immunoglobulin class switch recombination. $J$ Exp Med 2009; 206: 1073-1087.

32. Salier JP, Goust JM, Pandey JP, et al. Preferential synthesis of the G1m(1) allotype of $\operatorname{IgG1}$ in the central nervous system of multiple sclerosis patients. Science 1981; 213: 1400-1402.

33. Goust JM and Salier JP. Imbalance in recruitment of IgG (Gm) allotype-producing B-cell subsets from blood to brain in multiple sclerosis. Cell Immunol 1984; 88: 551-557.

34. International Multiple Sclerosis Genetics Consortium (IMSGC), Beecham AH, Patsopoulos NA, et al. Analysis of immune-related loci identifies 48 new susceptibility variants for multiple sclerosis. Nat Genet 2013; 45: 1353-1360. 